\title{
Dietary habits and the metabolic syndrome in middle-aged Europeans
}

\author{
H. Parr ${ }^{1}$, B. Stewart-Knox ${ }^{1}$, B. Bunting ${ }^{2}$, S. Pinhão ${ }^{3}$, M. D. V. de Almeida ${ }^{3}$ and M. J. Gibney ${ }^{4}$ \\ ${ }^{1}$ Northern Ireland Centre for Food \& Health and ${ }^{2}$ School of Psychology, University of Ulster, Coleraine, UK, \\ ${ }^{3}$ Faculty of Nutrition and Food Sciences, University of Porto, Porto, Portugal and ${ }^{4}$ University College Dublin, Dublin, \\ Republic of Ireland
}

The metabolic syndrome is a cluster of conditions (central obesity, elevated blood pressure, abnormal lipid profile and insulin resistance) associated with increased risk of developing CHD and/or diabetes. Metabolic syndrome may be dietary related ${ }^{(1)}$. The aim of the present analysis has been to explore associations between dietary habits, body fat distribution and patterns in the reporting of conditions related to metabolic syndrome in European consumers.

Data were collected from a representative sample aged $\geq 40$ years by survey $(n 1722)$ in Great Britain $(n 1182)$ and Portugal $(n 540)$ as part of the LIPGENE project. Dietary habits for major food groups were assessed using a short FFQ. Anthropometric measures were BMI and waist circumference (WC). FFQ data were factor analysed (maximum likelihood extraction, promax rotation; Kaiser normalised) and self-reported conditions associated with metabolic syndrome (high blood cholesterol, high blood pressure, mid-waist obesity, high or low blood sugar) underwent latent class analysis Mplus( version 4 software ${ }^{(2)}$.

Based on the factor analysis of FFQ, three clusters of dietary habits (Table 1) emerged that were characterised by: first, frequent alcohol consumption and infrequent intake of fruit and vegetables and other food groups (factor 1); second, infrequent alcohol consumption, but frequent intake of high-fat-containing foods (factor 2); third, frequent consumption of wine (but infrequent intake of other alcoholic beverages) and consumption of a variety of food groups including fish and low-fat-containing foods (factor 3). Four latent classes emerged from patterns of self-reported metabolic syndrome signs (Table 2). One-way ANOVA tests were conducted for each dietary group in relation to metabolic syndrome classes (Table 1$)$. The metabolic syndrome class were less likely to be in either the unhealthy $(P \leq 0.001)$ or alcohol $(P=0.023)$ dietary group than the overweight class; the metabolic syndrome class were also less likely to be in the unhealthy $(P \leq 0.001)$ or alcohol group $(P=0.007)$ than the healthy class.

Table 1. FFQ factor loadings, pattern matrix; ANOVA FFQ factor scores by latent class

\begin{tabular}{lcrr}
\hline Food group & Factor 1 & Factor 2 & Factor 3 \\
\hline Milk, cheese, yogurts & -0.220 & 0.396 & 0.151 \\
Meat, poultry, meat products & 0.099 & 0.097 & 0.244 \\
Fish and/or eggs & 0.030 & -0.112 & 0.490 \\
Bread and cereals (e.g. rice, pasta) & -0.015 & 0.295 & 0.280 \\
Sweets, cakes, chocolate, biscuits & 0.000068 & 0.544 & -0.091 \\
Spreads (e.g. butter, margarine, lard) & 0.060 & 0.667 & -0.015 \\
Vegetable oils (e.g. olive, sunflower) & -0.044 & 0.073 & 0.481 \\
Vegetables and fruit & -0.079 & 0.215 & 0.280 \\
Wine & 0.311 & -0.184 & 0.321 \\
Alcoholic (non-wine) & 0.998 & 0.064 & -0.047 \\
FFQ factor scores and latent class & & & \\
'Metabolic syndrome' $(n$ 1648, df 3; & F 2.7, & F 10.2, & F 0.359, \\
$\quad$ one-way ANOVA) & $P=0.042^{* *}$ & $P \leq 0.001 * *$ & $P=0.783$ \\
\hline
\end{tabular}

Differences between classes were significant: $* * P<0.01$.

Table 2. Latent class characteristics

\begin{tabular}{lrccc}
\hline Class & $n$ & $\%$ & BMI $\left(\mathrm{kg} / \mathrm{m}^{2}\right)$ & Waist circumference $(\mathrm{cm})$ \\
\hline 'Metabolic syndrome' & 378 & 22.0 & 29.9 & 97.9 \\
'Healthy' or no symptoms & 1006 & 58.4 & 24.9 & 84.8 \\
'Overweight' & 259 & 15.0 & 28.4 & 92.5 \\
'Obese' & 79 & 4.6 & 35.9 & 102 \\
\hline
\end{tabular}

Frequent alcohol consumption appears to be a marker for infrequent food consumption and infrequent alcohol a marker for intake of high-fat foods, while wine may be a marker for a healthy balanced diet. This theory requires further investigation.

Survey Fieldwork was sub-contracted to Ipsos MORI (GB). This work was completed on behalf of the LIPGENE Consortium and funded under the EU 6th Framework Food Quality and Safety Programme, code FOOD-CT-2003-505944.

1. Buttriss J \& Nugent A (2005) Proc Nutr Soc 64, 345-347.

2. Muthén BO \& Muthén LK (2006) MPlus Statistical Analysis with Latent Variables: User's Guide, 4th ed. Los Angeles, CA: Muthen \& Muthen. 